\title{
The Effects of Newly Formulated Filter Aids Material Loading on Pressure Drop and Particle Penetration
}

\author{
S. Hajar ${ }^{1, a}$, M. Rashid ${ }^{1, b,{ }^{*}, \text { A. Nurnadia }}{ }^{1, c}$ and M. R. Ammar ${ }^{2, d}$ \\ ${ }^{1}$ Air Resources Research Laboratory, Malaysia-Japan International Institute of Technology, \\ Universiti Teknologi Malaysia, 54100 Kuala Lumpur, Malaysia \\ ${ }^{2}$ AMR Environmental Sdn. Bhd., Taman Sri Pulai Perdana, 81110 Johor Bahru, Malaysia \\ ahajarahim@hotmail.com, brashidyusof.kl@utm.my, ${ }^{\mathrm{c}}$ nadyabit61@yahoo.com, \\ dammar_amrgroup@yahoo.com.my
}

Keywords: Filter aids, Activated carbon, PreKot ${ }^{\mathrm{TM}}$, Pressure drop, Particle penetration.

\begin{abstract}
Filter aids have been widely used in air filtration system in order to fulfill the needs to expand the lifespan of the fabric filter as well as to improve the collection efficiency of particles during filtration and separation operations. The efficiencies of PrekotAC that consists of a different combination of power activated carbon and pre-coating material, PreKot ${ }^{\mathrm{TM}}$ in terms of its pressure drop across a filter media and collection efficiency are highly influenced on the material loading of a material used during filtration process. PrekotAC was formulated into four different weight ratios and tested under a constant air flow rate of $4 \mathrm{~L} / \mathrm{min}$ and three different material loadings of $0.2,0.4$ and 0.6 $\mathrm{mg} / \mathrm{mm}^{2}$. It was found that the higher the material loading is the higher the pressure drop across a filter media becomes. The results also showed that the penetration of particles were reduced significantly as material loading increases where higher material loading leads to higher collection efficiencies of particles.
\end{abstract}

\section{Introduction}

Filter aids is an inorganic mineral powders or organic fibrous materials which is used together with filtration unit in order to improve and increase the efficiency of filtration and separation operations. It may be applied in various ways and pre-coat filter aids is one of the techniques which has been widely used in the industry where it will coat a layer of inert material onto the surface of the fabric filter. Filter aids has the ability to increase the porosity and reduce the compressibility of the cake thus make the accumulated cake more permeable. In filtration operations, pre-coat filter aids used to coat a layer of inert material onto the surface of the fabric filter which acts as a supporter for the accumulated cake as well as a barrier for protection that allow a uniform airflow passing through the fabric filter [1].

Filter quality is referring to the performance of the filter aids material in terms of its pressure drop as well as filtration efficiency since these two parameters are closely related to one another. A good filter aids should not only capable in extending the life span of the fabric filter but it should have the least number of particles penetrating through the filter media as well able to reduce pressure drop across a filter cake [2].

Pressure drop is made up of two components which are particle deposition within the filter media as well as the dust cake that formed on the surface of the filter media. The deposited dust that accumulated on the surface of the filter media is responsible for increasing the pressure drop across the filter media $[3,4]$. Pressure drop across the filter media as well as particle penetration depends on operation parameters such as filtration velocity, filter media (permeability and surface properties), characteristics of dust (composition, shape and particle size distribution), gas properties (composition and temperature) and parameters of bag cleaning that is pulse jet over pressure and duration [5, 6]. It was reported that filter cake thickness which referring to material loading could influenced the penetration performance of a material [7]. Thus, understanding the parameters effecting the performance of a filter aids material is important in order to find the best filter aids material that can be applied in the filtration and separation process. 
Hence, this paper discusses on the effects of material loading on the efficiency of PrekotAC in terms of its pressure drop and particle collection efficiency across a PTFE filter media. PrekotAC was tested in a laboratory scale filtration test system unit under a constant air flow rate of $4 \mathrm{~L} / \mathrm{min}$ and three different material loadings of $0.2,0.4$ and $0.6 \mathrm{mg} / \mathrm{mm}^{2}$. The performance of PrekotAC as a newly formulated filter aids material was studied and compared to the performance of its respective raw materials, activated carbon and PreKot ${ }^{\mathrm{TM}}$ in favor to find the suitable PrekotAC mixture that will be applied as filter aids material in fabric filtration system.

\section{Methodology}

Formulation of PrekotAC. PrekotAC is a combination of an adsorbent, activated carbon and a commercially available pre-coating material, PreKot ${ }^{\mathrm{TM}}$. Activated carbon used in this study is a coconut based material while PreKot ${ }^{\mathrm{TM}}$ is an amorphous hydrated glassy volcanic rock primarily fused aluminum silicate. The specifications of both raw materials have been summarized in Table 1 .

Table 1. Specifications of raw materials (activated carbon and PreKot ${ }^{\mathrm{TM}}$ ).

\begin{tabular}{c|c}
\hline Activated carbon & PreKot $^{\mathrm{TM}}$ \\
\hline Form, color: Powder, black & Form, color: Powder, snowy white \\
Origin: Coconut based & Fusion point: $1300-1400^{\circ} \mathrm{C}$ \\
pH: $9-11$ & Softening point: $900-1100^{\circ} \mathrm{C}$ \\
Ash content: $8 \%$ max & Thermal conductivity: Less than $0.0500\left(\mathrm{kcal} / \mathrm{mh}^{\circ} \mathrm{C}\right)$ at $0^{\circ} \mathrm{C}$ \\
Bulk density: $\sim 440 \mathrm{~kg} / \mathrm{m}$ & Bulk density: $\sim 120 \mathrm{~kg} / \mathrm{m}^{3}$ \\
\hline \multicolumn{2}{c}{}
\end{tabular}

PrekotAC was formulated into four different weight ratios as presented in Table 2. Activated carbon and PreKot ${ }^{\mathrm{TM}}$ were dried in an oven (Memmert, Model UNB 200) at $110^{\circ} \mathrm{C}$ for 24 hours before formulating in order to discard the moisture content.

Table 2. Proposed compositions of PrekotAC.

\begin{tabular}{c}
\hline Activated carbon: PreKot ${ }^{\mathrm{TM}}[$ Ratio (wt $\left.\%)\right]$ \\
\hline $10: 90$ \\
$20: 80$ \\
$30: 70$ \\
$40: 60$ \\
\hline
\end{tabular}

\section{Experimental Procedures.}

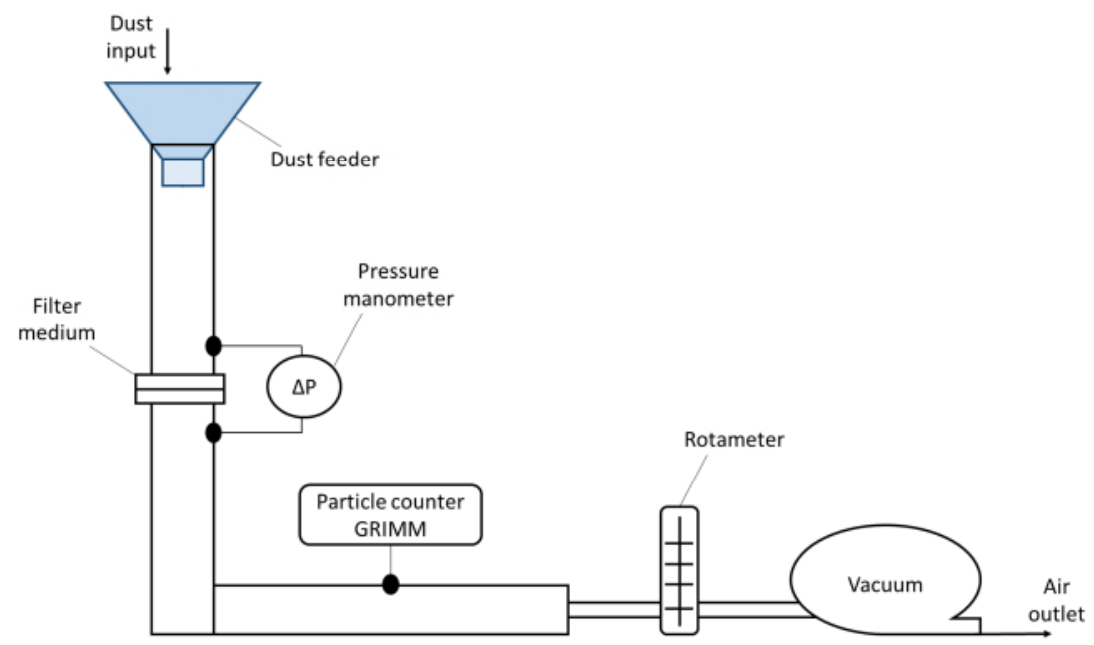

Fig. 1 Schematic diagram of the filtration test system. 
Fig. 1 showed the laboratory scale filtration test system that had been designed to study and evaluate the performance of each formulated filter aids materials as well as the raw materials. The fabricated test system consisted of dust feeder, filter media holder, pressure manometer, rotameter, particle counter and a vacuum pump. Pressure drop across a filter media was monitored using pressure manometer (Extech Instrument Model HD755) while particle penetration was observed using GRIMM Portable Laser Aerosol Spectrometer (Model 1.109). An air vacuum was placed at the end of the system to control the volumetric airflow rate that passing through the system.

Using the filtration test system as shown in Fig. 1, the effects of material loading on the efficiency of PrekotAC as a filter aids material under a constant air flow rate of $4 \mathrm{~L} / \mathrm{min}$ were investigated. The experiment was tested on PTFE filter media where the specifications of the filter media are presented in Table 3. The overall experimental procedures involved in this study are summarized in Table 4.

Table 3. Specifications of PTFE filter media.

\begin{tabular}{c|c}
\hline Basic weight $\left(\mathrm{g} / \mathrm{m}^{2}\right)$ & 800 \\
Thickness $(\mathrm{mm})$ & 1.3 \\
Working temperature $\left({ }^{\circ} \mathrm{C}\right)$ & $240-260$ \\
Breaking strength $(\mathrm{N} / 5 \mathrm{~cm})$ & Wrap $\geq 700$, Weft $\geq 700$ \\
\hline Table 4. Summary of the experimental procedures. \\
\hline Filter media & PTFE \\
Total filtration area $\left(\mathrm{mm}^{2}\right)$ & 755 \\
Material loading $\left(\mathrm{mg} / \mathrm{mm}^{2}\right)$ & $0.2,0.4,0.6$ \\
Airflow rate $(\mathrm{L} / \mathrm{min})$ & 4 \\
\hline
\end{tabular}

\section{Results and Discussions}

Effects on Pressure Drop Across a Filter Media. Fig. 2 presents the effects of different material loading of $0.2,0.4$ and $0.6 \mathrm{mg} / \mathrm{mm}^{2}$ on pressure drop across a filter media under constant airflow rate of $4 \mathrm{~L} / \mathrm{min}$ which as expected showed that pressure drop across the filter cake increases with material loading for all materials. As shown in the figure, each material registered the highest pressure drop across the filter cake at the highest material loading of $0.6 \mathrm{mg} / \mathrm{mm}^{2}$. This can be explained by the fact that higher material loading leads to a thicker filter cake and consequently reduces the volumetric air flow that passing through the filter cake [8]. This finding indicates the dependence of pressure drop on the permeability of the accumulated dust cake on the filter media $[9,10]$.

As in Fig. 2, activated carbon has the highest while PreKot ${ }^{\mathrm{TM}}$ presents the lowest pressure drop across the filter cake in all cases. PrekotAC showed a consistent and uniform pressure drop across a filter cake with PrekotAC 10:90 has the highest while PrekotAC 40:60 registered the lowest pressure drop among these four formulations. It was observed that the addition of $\operatorname{PreKot}^{\mathrm{TM}}$ to the formulation gave a lower pressure drop across a filter cake compared to activated carbon alone. It was found that the porous and fluffy structure of PreKot ${ }^{\mathrm{TM}}$ help to form a porous filter cake that allows a uniform airflow passing through the filter media with low pressure drop despite of its thickness. On contrary, activated carbon which mainly consists of fine particles has a higher tendency to form a compacted filter cake which reduces airflow passing through the filter media resulting in higher pressure drop across the filter cake $[11,12]$. 


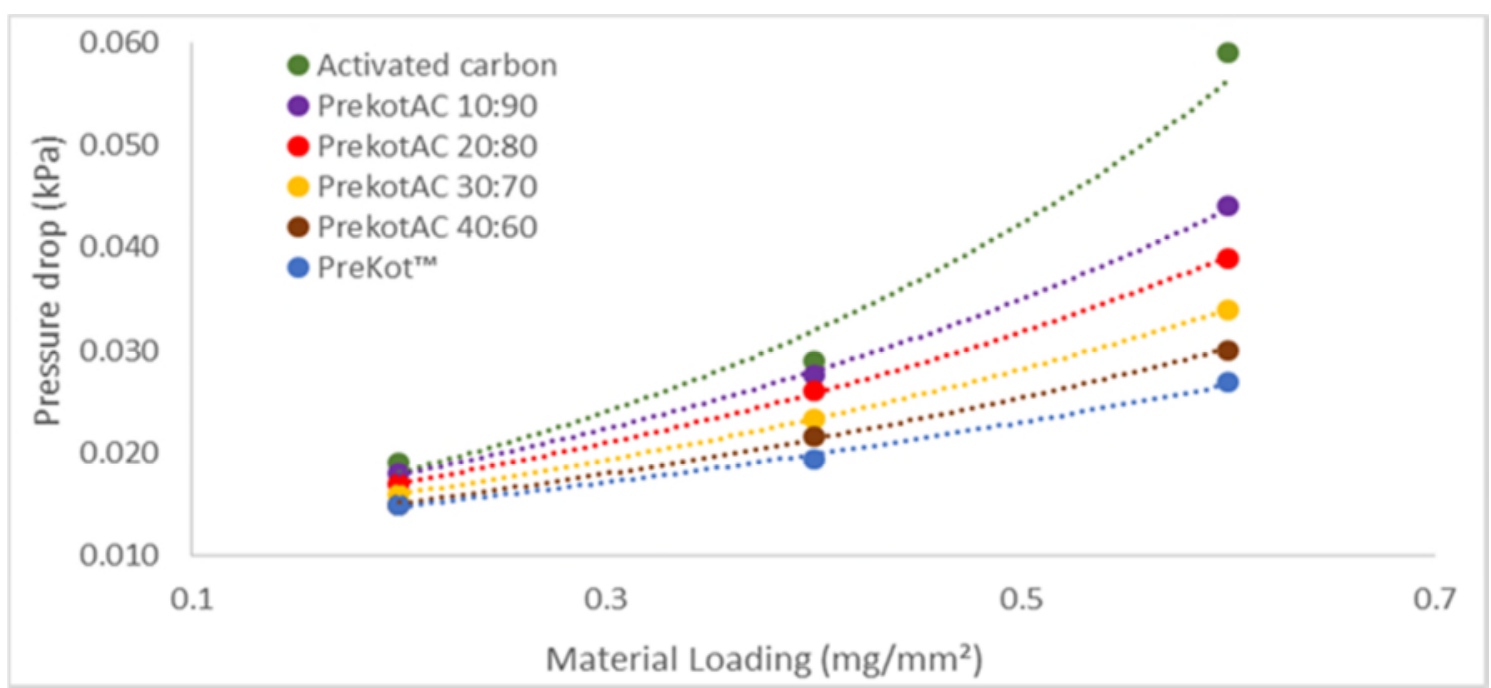

Fig. 2 Effects of material loading on pressure drop across a filter media under a constant airflow rate of $4 \mathrm{~L} / \mathrm{min}$.

Thus, it concludes that pressure drop across the filter cake is affected by porosity of the filter cake that accumulated on the filter media. The porosity of the filter cake decreases as the material loading increases because higher material loading leads to higher filter cake thickness and compression stress on the filter cake. However, the newly formulated mixture PrekotAC materials still exhibit the lower pressure drop compared to the original material activated carbon even at the highest material loading of $0.6 \mathrm{mg} / \mathrm{mm}^{2}$. The characteristics of the mixture itself which has wide disparities particle size distribution leads to form a porous filter cake with higher permeability and lower pressure drop across a filter cake even at the highest material loading.

Effects on Particle Penetration. The performance in terms of particle penetration was calculated based on Eq. (1), which is the ratio of the number of penetrated ambient particles across the blank filter over the number of penetrated filter aids particles under various material loadings [13].

Ratio of penetrated particles $(\mathrm{Rpp})=\mathrm{Tf} / \mathrm{Ti}$ where;

$\mathrm{R}_{\mathrm{pp}}=$ ratio of the number of penetrated particles

$\mathrm{T}_{\mathrm{f}}=$ total number of penetrated particles after filter aids was added

$\mathrm{T}_{\mathrm{i}}=$ total number of penetrated particles for ambient air particles

The performance of each material was evaluated based on the calculated $\mathrm{R}_{\mathrm{pp}}$ value where;

1 = initially, without material loading

I.e. the ratio of the number of penetrated particles for fabric filter alone, based on ambient air particle

$>1=$ better filtration efficiency, less penetration compared to fabric filter alone

$>1=$ poor filtration efficiency, higher penetration compared to fabric filter alone

Fig. 3 presents the ratio of the number of penetrated particles through a PTFE filter media, with different amount of filter aids loadings of $0.2,0.4$ and $0.6 \mathrm{mg} / \mathrm{mm}^{2}$ under a constant airflow rate of 4 $\mathrm{L} / \mathrm{min}$ which that the activated carbon retains the highest while PreKot ${ }^{\mathrm{TM}}$ has the lowest number of penetrated particles across the filter media in all cases. As stated earlier, activated carbon which predominantly consists of smaller particles has higher number of penetrated particles ratio compared to PreKot ${ }^{\mathrm{TM}}$. It was reported that coarser particles have higher collection compared to finer particles. Thus, as expected, activated carbon has higher amount of penetrated particles compared to the other filter aids material that has coarser particle sizes $[14,15]$. 


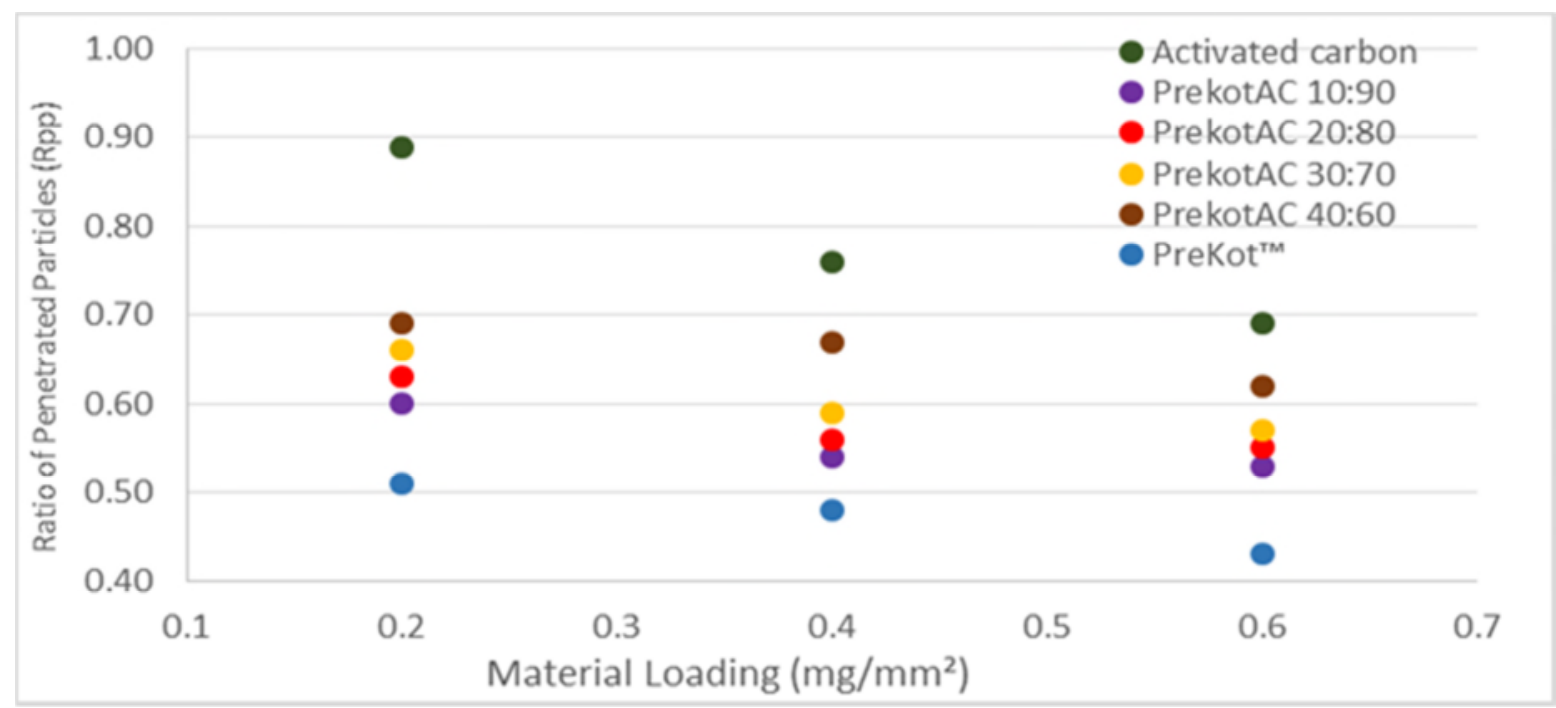

Fig. 3 Effects of material loading on particle penetration under constant airflow rate of $4 \mathrm{~L} / \mathrm{min}$.

As in the finding, PrekotAC 40:60 has the highest while PrekotAC 10:90 registered the lowest total number of penetrated particles in all cases. It was found that the addition of PreKot ${ }^{\mathrm{TM}}$ to activated carbon gave a better collection efficiency compared to the performance of activated carbon alone. It seems that PreKot ${ }^{\mathrm{TM}}$ acts as and additional filter media and helps to block the fine particles from penetrating through it.

It was expected that PrekotAC 40:60 which has a higher percentage of coarse particle will give a better collection efficiency with less particle penetration compared to PrekotAC 10:90. However, the results obtained were completely different to the preliminary prediction. It was found that the higher the ratio of PreKot ${ }^{\mathrm{TM}}$ in the mixture is, the bigger the total particle penetration becomes. This occurred because of the effect of the characteristics of PreKot ${ }^{\mathrm{TM}}$ itself which has an odd ball shapes of a loosely pack material with larger size particles and porosity, leads to higher total particle penetration since fine particles can easily penetrating through it [16].

However, as the material loading increases, the ratio of the number of penetrated particles decreases in all cases. As in Fig. 3, as the material loading increased to $0.6 \mathrm{mg} / \mathrm{mm}^{2}$, all filter aids material registered a better collection efficiency with the $R_{p p}$ value of less than 1.0. The total penetration of each material is following the trend that was reported by previous researcher which is higher material loading leads to a lower total particle penetration due to its thicker cake thickness that avoid and block particles from penetrating through the filter media $[17,18]$.

Thus, based on the results obtained, it can be concluded that filtration efficiency is highly dependent on the material loading introduced during filtration process where the higher the material loading is, the lower the total number of particles that penetrating through the filter media becomes.

\section{Conclusion}

The performance a filter aids material is highly dependent to the material loading introduced during the filtration process. It was reported that higher material loading leads to higher pressure drop due across a filter cake formation. This occurred because thicker filter cake reduces the volumetric air flow passing through the media resulting in higher pressure drop across it. However, in term of its particle penetration, the higher the material loading is, the lower the ratio of penetrated particles becomes presenting a higher collection efficiency. This is due to a lower material loading gives loosely packed particles that easily permeate through the filter media. However, at a higher material loading, the compacted filter cake reduces the total particle penetration across the filter aids material. The study concludes that the performance of the filter aids material is strongly influenced by the material loading introduced during the filtration and separation process. In addition, PrekotAC showed a better performance compared to the raw material activated carbon even at the highest material loading. 


\section{References}

[1] T. Barnett, Improving the Performance of Fabric Filter Dry Dust Collection Equipment. Filtr. Separat. 37(2) (2000) 28-32.

[2] K. M. Lee, Y. M. Jo, J. H. Lee and J. A. Raper, Assessment of Surface and Depth Filters by Filter Quality. Powder Technol. 185 (2008) 187-194.

[3] M. Saleem and G. Krammer, Effect of Filtration Velocity and Dust Concentration on Cake Formation and Filter Operation in a Pilot Scale Jet Pulsed Bag Filter. J. Hazard. Mater. 144 (2008) 677-681.

[4] Y. Endo, D. R. Chen and D. Y. H. Pui, Effects of Particle Polydispersity and Shape Factor during Dust Cake Loading on Air Filters. Powder Technol. 98 (1997) 241-249.

[5] M. Saleem, G. Krammer, R. U. Khan and M. S. Tahir, Influence of Operating Parameters on Cake Formation in Pilot Scale Pulse-jet Bag Filter. Powder Technol. 224 (2012) 28-35.

[6] L. K. Wang, N. C. Pereira and Y. T. Hung, Air Pollution Control Engineering. Humana Press. (2004) 59-95.

[7] D. Leith and M. W. First, Performance of a Pulse-jet Filter at High Filtration Velocity, I. Particle Collection. Japca. J. Air Waste. Ma. 27(6) (1977) 534-539.

[8] K. J. Jeon and Y. W. Jung, A Simulation Study on the Compression Behaviour of Dust Cakes. Powder Technol. 141 (2004) 1-11.

[9] H. Leibold and J. G. Wilhelm, Investigations into the Penetration and Pressure Drop of HEPA Filter Media during Loading with Submicron Particle Aerosols at High Concentrations. J. Aerosol Sci. 22 (1991) 773-776.

[10] C. Kanaoka, and M. Amornkitbamrung, Effect of Filter Permeability on the Release of Captured Dust from a Rigid Ceramic Filter Surface. Powder Technol. 118 (2001) 113-120.

[11] S. Hajar, M. Rashid, A. Nurnadia, H. Norelyza and M. Ammar, PrekotAC as Filter Aids for Efficient Dust Separation in a Fabric Filter. Jurnal Teknologi. 67(4) (2014) 29-31.

[12] A. Nurnadia, M. Rashid, S. Hajar and M. Ammar, Effect of Filter Aids on Pressure Drop and Permeability of Two Different Filter Media. Adv. Mater. Res. 1125 (2015) 245-249.

[13] A. Nurnadia, M. Rashid, S. Hajar and M. Ammar, Penetration of Particles across a Newly Formulated Filter Aids Material. Presented at 3rd International Science Postgraduate Conference 2015, Johor Bahru, Malaysia.

[14] M. D. M. Innocentini, V. P. Rodrigues, R. C. O. Romano, R. G. Pileggi, G. M. C. Silva and J. R. Coury, Permeability Optimization and Performance Evaluation of Hot Aerosols Filters Made Using Foam Incorporated Alumina Suspension. J. Hazard. Mater. 162 (2009) 212-221.

[15] K. M. Lee, Y. M. Jo, J. H. Lee and J. A. Raper, Assessment of Surface and Depth Filters by Filter Quality. Powder Technol. 185 (2008) 187-194.

[16] S. Hajar, M. Rashid, A. Nurnadia and M. R. Ammar, PrekotAC as a New Filter Aids Material for Fabric Filter in Air Filtration System. Jurnal Teknologi. 72 (2015) 1-6.

[17] B. H. Park, S. B. Kim, Y. M. Jo and M. H. Lee, Filtration Characteristics of Fine Particulate Matters in a PTFE/Glass Composite Bag Filter. Aerosol Air Qual. 12 (2012) 1030-1036.

[18] C. B. Song, H. S. Park and K. W. Lee, Experimental Study of Filter Clogging with Monodisperse PSL Particles. Powder Technol. 163 (2006) 152-159. 